\title{
Kundenorientierte strategische Auswahl von Geschäftsmodellen für IoT-Ökosysteme
}

Die Digitalisierung erfordert von Unternehmen eine weitaus stärkere Kundenorientierung als in der Vergangenheit. Gleichzeitig werden insbesondere Produkte und Dienstleistungen mit Bezug zum Internet of Things (IoT) in der Regel über Plattformen und Ökosysteme betrieben und vermarket. All dies erfordert neue, strategisch abgestimmte Geschäftsmodelle. Auf Basis einer empirischen Studie zeigen wir auf, welche Geschäftsmodelle aus einer Kundenperspektive akzeptabel sind, und bieten Managern die Möglichkeit, diese Erkenntnisse direkt in die Praxis umzusetzen.

Michael Kubach und Cristina Mihale-Wilson

Wirtschaftsinformatik \& Management 2021 • 13 (4): 294-301 https://doi.org/10.1365/s35764-021-00330-1

Angenommen: 24. Januar 2021

Online publiziert: 8. April 2021

c c Der/die Autor(en) 2021 
Die Bedeutung von Geschäftsmodellen für den langfristigen Unternehmenserfolg ist unbestreitbar. Sie definieren, wie Unternehmen Wert schaffen und den Kunden bereitstellen sowie Profite für Unternehmen generieren. Darüber hinaus spiegeln sie die Hypothese des Managements darüber wider, was Kunden wollen, wie sie es wollen und wie das Unternehmen organisiert werden kann, um diese Bedürfnisse am besten zu erfüllen [1].

Die Digitalisierung und die damit verbundenen Chancen und Risiken zwingen Unternehmen, ihre Geschäftsmodelle zu überdenken und/oder bestehende Geschäftspraktiken zu innovieren. Um die Herausforderungen der digitalen Wirtschaft zu meistern, müssen Manager geeignete Strategien finden, um die Interessen des Unternehmens und ihrer Kunden zu berücksichtigen, ohne dabei das Wertschöpfungspotenzial des Unternehmens oder das Vertrauen der Kunden zu gefährden. Dies ist eine besonders herausfordernde Aufgabe, da Unternehmen in einem Spannungsfeld zwischen Chancen und Herausforderungen der Digitalisierung agieren, wobei sie nur über begrenzte Ressourcen verfügen können. Als erschwerende Rahmenbedingungen kommen die begrenzte Zahlungsbereitschaft der Endkunden für bestimmte Produkte und Dienstleistungen und die Notwendigkeit, das Vertrauen der Endkunden zu gewinnen und zu erhalten, hinzu.

Eine zentrale Herausforderung, die sich aus der Digitalisierung ergibt, sind die gegensätzlichen Interessen von Unternehmen und Kunden, wenn es um die Erhebung und Verarbeitung von Kundendaten geht. Während Unternehmen Daten als kritischen Produktionsfaktor für die Wertschöpfung sehen und deshalb große Datenbestände aufbauen möchten, wollen Kunden tendenziell die Kontrolle über ihre Daten behalten und diese schützen. Hiermit verbunden ist der Wunsch der Kunden nach Transparenz und Wissen darüber, wo und wie ihre Daten verwendet werden. Das Umfeld von IoT-Produkten und -Dienstleistungen eignet sich dabei besonders gut zur Verdeutlichung dieses zunehmenden Konfliktes zwischen dem Bestreben von Unternehmen zur Wertschöpfung aus Kundendaten und dem Wunsch der Endkunden nach Transparenz und Datensouveränität. Zeichnen sich doch gerade IoT-Geschäftsmodelle für Plattformen und Ökosysteme durch den engen Einbezug der Endkunden in den Wertschöpfungsprozess, Serviceorientierung und die Analyse der Vielzahl anfallender Daten aus.

Insbesondere in der Konkurrenz zu internationalen Plattformkonzernen mit großen finanziellen und technologischen Ressourcen sowie Möglichkeiten zum „kreativen“ Umgang

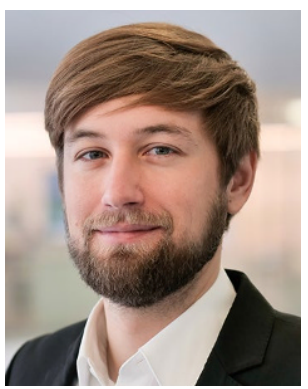

Dr. Michael Kubach ${ }^{1}(\triangle)$

befasst sich am Fraunhofer-Institut für Arbeitswirtschaft und Organisation IAO in internationalen und nationalen Forschungs- sowie Industrieprojekten mit ökonomischen Fragen im weiteren Umfeld von IT-Sicherheit und digitalen Identitäten.

michael.kubach@iao.fraunhofer.de

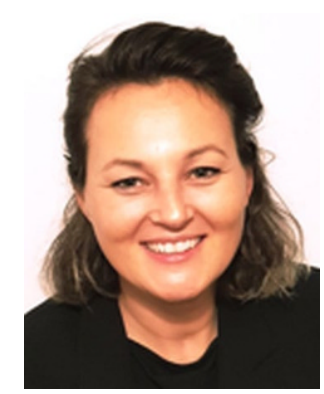

Cristina Mihale-Wilson ${ }^{2}(\triangle)$

beschäftigt sich an der Goethe-Universität Frankfurt am Main hauptsächlich mit ökonomischen und sozialen Aspekten sowie Potenzialen künstlicher Intelligenz und digitaler Ökosystemen.

mihale-wilson@wiwi.uni-frankfurt.de

${ }^{1}$ Fraunhofer IAO,

Stuttgart/Berlin, Deutschland

${ }^{2}$ Goethe-Universität,

Frankfurt am Main, Deutschland

mit Datenschutzvorgaben, wie etwa durch die EU-Datenschutzgrundverordnung (DSGVO), sind die europäischen Unternehmen gezwungen, eine strategisch abgestimmte Neuausrichtung ihrer Geschäftsmodelle vorzunehmen. Doch wie können Unternehmen bei der Auswahl und Gestaltung ihrer (neuen) Geschäftsmodelle für IoT-Ökosysteme konkret 


\section{Zusammenfassung}

- Die zunehmende Bedeutung von Plattformen und Ökosystemen mit den damit verbundenen Chancen und Risiken zwingen Unternehmen umzudenken und ihre Geschäftsmodelle zu innovieren.

- Um die Herausforderungen der digitalen Wirtschaft zu meistern, müssen Unternehmen geeignete Strategien finden, um ihre eigenen Interessen und die ihrer Kunden zu berücksichtigen, ohne dabei das Wertschöpfungspotenzial des Unternehmens oder das Vertrauen der Kunden zu gefährden.

- Unter der Prämisse, dass eine Berücksichtigung der Kundenbedürfnisse und -präferenzen in der Geschäftsmodellauswahl der Unternehmen eine nachhaltig erfolgreiche Unternehmensstrategie sein kann, bietet dieser Beitrag praxisrelevante Hinweise, die vor allem für kleine und mittelständische Unternehmen wertvoll sein können.

vorgehen, wovon können sie sich leiten lassen? Welche Geschäftsmodelle eignen sich besonders gut, um einerseits den Unternehmenserfolg nachhaltig zu sichern und andererseits den Kundenpräferenzen hinsichtlich des Umgangs mit ihren Daten gerecht zu werden?

Vor diesem Hintergrund bietet der Beitrag im Folgenden eine Orientierungshilfe für Akteure, die vor der Herausforderung stehen, nachhaltige und für die Teilnahme an digitalen Plattformen und Ökosystemen passende Geschäftsmodelle zu etablieren. Insbesondere kleinen und mittelständischen Unternehmen stehen in der Regel nur limitierte Kompetenzen im Bereich der strategischen Geschäftsmodellentwicklung zur Verfügung. Unser Vorgehen setzt hier an. Es identifiziert Geschäftsmodelle, die die Kundenperspektive widerspiegeln, dadurch das Kundenvertrauen fördern und deshalb potenziell nachhaltiger sind als die aktuell häufig propagierten Alternativen, die hauptsächlich auf der Monetarisierung von Kundendaten basieren.

\section{Relevanz von Kundenperspektive und Vertrauen für Geschäftsmodelle}

Die Monetarisierung von und Wertschöpfung aus Kundendaten erscheinen zunächst als eindeutig sinnvolle Geschäftsmodellelemente in IoT-Ökosystemen. Unternehmen analysieren Kundendaten, um den Vertrieb ihrer Produkte zu verbessern,
Suchkosten zu reduzieren, Transaktionskosten zu senken, Preisdiskriminierung zu betreiben, Produkte zu individualisieren und so weiterzuentwickeln, dass sie einen Preisaufschlag verlangen können. Diese Strategien werden entsprechend auch von vielen Unternehmen verfolgt - Daten gelten als das „neue Öl“. Dennoch können hieraus auch Gefahren für das langfristige Kundenvertrauen und dementsprechend die Nachhaltigkeit dieser Geschäftsmodelle erwachsen. Die wahrgenommene Bedrohung eines Geschäftsmodelles für die Privatsphäre der Kunden spielt für diese durchaus eine Rolle für ihre Konsumentscheidungen. Die Beziehung zwischen der Nutzung von Kundendaten, dem hieraus erwachsenden tatsächlichen Eingriff in die Privatsphäre und dem Kundenvertrauen bzw. der Akzeptanz durch Kunden ist dabei allerdings komplex. So zeigt sich in Studien etwa, dass Kunden der Nutzung ihrer Daten durch intelligente Assistenzsysteme skeptisch gegenüberstehen, wenn sie direkt nach ihrer Einwilligung hierzu gefragt werden. Zeitgleich akzeptieren Kunden, dass ihnen personalisierte Anzeigen ohne eine explizite Einwilligung zur Datenverarbeitung angezeigt werden $[2,3]$. Vor dem Hintergrund, dass Kunden zunehmend sensibler auf die Nutzung ihrer Daten reagieren und Missbrauchsfälle breit diskutiert werden, werden sich voraussichtlich auf lange Sicht nur die Geschäftspraktiken etablieren, mit denen die Verbraucher einverstanden sind.

Angesichts der gut dokumentierten Bedenken der Endkunden gegenüber der unbefugten und/oder intransparenten Sammlung und Verarbeitung ihrer Daten vertreten wir die Überzeugung, dass Unternehmen das Vertrauen der Endkunden gewinnen können, indem sie deren Bedürfnisse und Präferenzen auch in ihre strategischen Geschäftsmodellentscheidungen mit einbeziehen. Eine strategische Geschäftsmodellentscheidung, welche die Kundenpräferenzen und Bedürfnisse widerspiegelt, setzt ein wichtiges positives Signal für die Endkunden. Darüber hinaus verfügt sie über das Potenzial zur positiven Beeinflussung der Wahrnehmung des Unternehmens und seiner Produkte und Dienstleistungen.

\section{Geschäftsmodellmuster als Bausteine innovativer Geschäftsmodelle}

In der Wissenschaft wie auch in der managementorientierten Literatur existieren zahlreiche Ansätze zur Gliederung und Strukturierung von Geschäftsmodellen. Eine aufschlussreiche Taxonomie für Geschäftsmodelle wurde von Gassmann und seinem Team an der Universität St. Gallen erarbeitet [4]. Diese wurde auf der Grundlage einer umfassenden Analyse 
von zunächst 250 Geschäftsmodellen entwickelt, die in den letzten 25 Jahren in verschiedenen Branchen und Geschäftskontexten erfolgreich umgesetzt wurden. Laut dieser Analyse sind $90 \%$ der 250 Geschäftsmodelle aus der Rekombination von Konzepten entstanden. Hierauf aufbauend identifizierten die Autoren 55 Geschäftsmodellmuster, die den Kern vieler Geschäftsmodelle darstellen. Diese resultierende Liste von Geschäftsmodellmustern erscheint damit nicht nur als einer der jüngsten und umfassendsten Versuche, Geschäftsmodelle zu klassifizieren und zu identifizieren, sondern auch als sehr praxistauglich und anschaulich aufbereitet. Die 55 Geschäftsmodellmuster wurden nach einer transparenten und nachvollziehbaren Methodik erarbeitet. Sie reduzieren die Komplexität, was eine weitergehende Untersuchung ermöglicht, während sich gleichzeitig durch ihre Rekombination praktisch unbegrenzt neue Geschäftsmodelle entwickeln lassen.

\section{Studien: Von Kunden präferierte Geschäfts- modellmuster}

Zur Untersuchung, welche Geschäftsmodelle Kunden in IoTÖkosystemen präferieren, wurde schließlich eine zweistufige wissenschaftliche Studie durchgeführt. Diese kombinierte die Erkenntnisse über Geschäftsmodelle aus verwandten wissenschaftlichen Arbeiten in einer Expertenbefragung mit einer groß angelegten empirischen Untersuchung.

Als Ausgangspunkt dienten die oben genannten $55 \mathrm{Ge}$ schäftsmodellmuster nach Gassmann et al. [4]. Nicht alle 55 Geschäftsmodellmuster sind jedoch von Relevanz für di-

\section{Handlungsempfehlungen}

- Unternehmen, die eine Neuorientierung ihrer bestehenden Distributionsstrategie - insbesondere auch für IoT-Ökosysteme - vornehmen möchten, sollten das Supermarkt- sowie das Direktvertriebsmodell in Betracht ziehen.

- Hinsichtlich der Frage, ob Produkte und Dienstleistungen individualisierbar sein sollen, sollten Unternehmen Mass Customization oder No-Frills-Modelle in Betracht ziehen. Preisaufschläge werden von den Kunden allerdings nur bei individualisierbaren Produkten und Dienstleistungen akzeptiert.

- Bezüglich geeigneter Preisstrategien bevorzugen Kunden die Flatrate-, Freemium- oder Cross-SellingStrategie. gitale IoT-Ökosysteme. Deshalb wurden fünf Experten mit unterschiedlichem Erfahrungshintergrund (akademische Wissenschaft, praxisorientierte Forschung, Industrie) aus verschiedenen Branchen (z. B. Automotive, Consulting, Telekommunikation) gebeten, die Geschäftsmodellmuster hinsichtlich ihrer grundsätzlichen Eignung für digitale IoT-Ökosysteme zu bewerten. Die Bewertungen erfolgten zunächst getrennt. Anschließend fand ein Klärungsworkshop statt, um besonders herausragende Diskrepanzen in der Bewertung aufzulösen.

Aus den 55 Geschäftsmodellmustern identifizierten die Experten insgesamt 18 als relevant für die weitere Untersuchung (siehe Tab. 1). Die 18 ausgewählten Geschäftsmodellmuster wurden wiederum basierend auf ihrer Zugehörigkeit zum Marketingmix ${ }^{1}$ in vier Gruppen geclustert. Distribution (vier Geschäftsmodellmuster), Individualisierung (drei Geschäftsmodellmuster), Preisaufschläge (vier Geschäftsmodellmuster) und Sonstige Preisstrategien (neun Geschäftsmodellmuster). Das Geschäftsmodellmuster Mass Customization wurde in zwei Cluster einsortiert.

Im nächsten Schritt wurde eine Nutzerbefragung mit 301 Personen durchgeführt. Methodisch nutzte die Befragung das zur Präferenzerhebung hinsichtlich der relativen Bewertung bestimmter Attribute besonders geeignete BestWorst-Choice-Verfahren (Case 1) nach Flynn et al. [6]. Bei dieser Methode wählen die Teilnehmer aus jeweils drei auf einmal gezeigten Geschäftsmodellmustern das von ihnen am meisten und am wenigsten bevorzugte aus. Um sicherzustellen, dass alle Teilnehmer die abgefragten Geschäftsmodellmuster und deren Implikationen verstehen, wurden die von Gassmann et al. [4] formulierten Geschäftsmodellmuster in eine für Endkunden verständliche Aussage für den Kontext unserer Untersuchung „übersetzt“. Beispielsweise wurde für das Supermarket-Geschäftsmodellmuster den Studienteil-

1 Der Marketingmix umfasst alle Handlungen und Entscheidungen, die für eine erfolgreiche Platzierung eines Unternehmens und seiner Produkte und/oder Dienstleistungen auf dem Markt von Bedeutung sind. Er besteht aus vier Säulen den 4P [5]: Product (Produktpolitik)/Price (Preispolitik)/ Place (Distributionspolitik)/Promotion (Kommunikationspolitik). Für diese Studie wurde die Kommunikationspolitik außer Acht gelassen. Sie ist ein wichtiges Instrument, spiegelt sich jedoch nicht direkt in den 55 Geschäftsmodellmustern wider. 
nehmern folgende Aussage präsentiert: „Ich wähle meine Apps und Assistenzdienste auf einem zentralen Marktplatz aus“. Die „Übersetzung“ der Geschäftsmodellmuster in für Endkunden verständliche Aussagen wurde in einem Pre-Test überprüft. Die Befragung wurde in Zusammenarbeit mit einem deutschen Marktforschungsinstitut durchgeführt, das eine repräsentative Stichprobe von deutschen Teilnehmern zur Verfügung stellte.

Durch die Auswahl der von ihnen am meisten und am wenigsten bevorzugten Geschäftsmodellmuster aus den ihnen präsentierten Geschäftsmodellmustergruppen geben die Teilnehmer ihre Präferenzen preis. Hierauf aufbauend lässt sich dann ein entsprechendes Ranking für Geschäftsmodellmuster aus der Kundenperspektive erstellen. Dieses ist in Tab. 2 wiedergegeben. Dort ist darüber hinaus auch eine Bewertung ein „Score“ (skaliert zwischen 100 und 0) für jedes der $18 \mathrm{ab-}$ gefragten Geschäftsmodellmuster aufgeführt. Dies ermöglicht uns, die Präferenzwerte für jedes Geschäftsmodell im Verhältnis zu den anderen im jeweiligen Geschäftsmodellmustercluster zu analysieren.

\section{Tab. 1 Überblick über relevante Geschäftsmodellmuster}

\section{Cluster}

Distribution

Direct Selling

Supermarket

Full-Service-Provider

Individualisierung

Ultimate Luxury

Mass Customization

No Frills

Preisaufschläge

Guaranteed Availability

Robin Hood

Solution Provider

Mass Customization

Sonstige Preisstra- Freemium tegien

Add-on

Cross-Selling

Subscription

Flatrate

Pay-per-Use

Barter

Hidden Revenue

Self-Service
Produktvertrieb direkt an den Verbraucher (z. B. Download via Unternehmenshomepage)

Das Unternehmen vertreibt Apps und Dienste über einen App-Store, zusammen mit einer Vielzahl von Produkten anderer Unternehmen

Das Unternehmen vertreibt eine Gesamtabdeckung von Apps und Diensten, die in einem firmeneigenen App-Store zusammengefasst sind

Apps und Dienste haben einen sehr hohen Qualitätsstandard oder bieten exklusive Privilegien und zielen auf das Luxussegment ab. Die Preise sind entsprechend hoch

Obwohl sie in Massenproduktionsumgebungen hergestellt werden, können Apps und Dienste die individuellen Bedürfnisse der Verbraucher erfüllen, zum Beispiel durch Modularisierung

Apps und Dienste werden so einfach wie möglich gehalten und konzentrieren sich auf die Bereitstellung eines Hauptnutzenversprechens

App oder Dienst ist garantiert ohne Ausfallzeit verfügbar

Das gleiche Produkt wird an zahlungskräftige Kunden zu einem viel höheren Preis verkauft als an weniger zahlungskräftige - hauptsächlich, um das Image des Unternehmens zu verbessern

Alle Produkte stammen aus einer Hand und sind somit perfekt aufeinander abgestimmt

Modularisierung von Apps und Diensten, sodass Einzelpersonen ihre Bedürfnisse durch Kombination einzelner Komponenten zu einem Produkt erfüllen können

Basisversionen von Apps und Diensten werden kostenlos abgegeben, während für eine erweiterte Premiumversion des Produkts bezahlt werden kann

Basisversionen von Apps und Diensten sind preisgünstig. Zusätzliche Funktionalitäten können in Stufen hinzugekauft werden. Diese Käufe treiben den Endpreis des Produkts in die Höhe

Zusätzliche Umsätze werden durch die Bereitstellung weiterer Produkte und Dienstleistungen erzielt, die nicht unbedingt direkt mit dem Hauptprodukt zusammenhängen

Der Verbraucher zahlt eine regelmäßige Gebühr (z. B. monatlich)

Der Verbraucher zahlt eine einzige feste Gebühr und kann für eine bestimmte Zeit eine Vielzahl von Produkten und Dienstleistungen unbegrenzt nutzen

Der Verbraucher zahlt nur für das, was er verbraucht

Obwohl kein tatsächliches Geld im Spiel ist, bezieht sich dieses Geschäftsmodell auf einen Austausch. Es wird dem Verbraucher z. B. eine App oder ein Dienst zur Verfügung gestellt, der Verbraucher liefert im Gegenzug bestimmte Informationen oder erfüllt Aufgaben

Haupteinnahmequelle des Unternehmens ist eine dritte Partei und nicht der Verbraucher

Ein Teil der Wertschöpfung wird an den Verbraucher übertragen, im Austausch für einen niedrigeren Preis 
Die Ergebnisse für den Cluster Distribution deuten darauf hin, dass Kunden es vorziehen, IoT-Produkte und -Dienstleistungen von verschiedenen Firmen zu nutzen, anstatt an ein einziges Unternehmen gebunden zu sein (Full-Service-Provider). Dass die Werte für das Supermarkt- und das Direktvertriebsmodell nicht allzu weit auseinanderliegen, deutet darauf hin, dass Kunden beide Geschäftsmodellmuster ähnlich stark präferieren. Somit können beide Geschäftsmodellmuster eine strategisch geeignete Wahl für die Distribution von Produkten und Dienstleistungen darstellen. Dementsprechend wäre für Manager bei ihrer endgültigen Entscheidung abzuwägen, ob ihr Unternehmen über die notwendigen Ressourcen verfügt, um einen Direktvertriebskanal realisieren zu können, oder ob sie lieber im Rahmen einer Supermarkt-Strategie mit passenden Unternehmen und Plattformen kollaborieren möchten.

Hinsichtlich des Clusters Individualisierung zeigen die Ergebnisse, dass Kunden modular aufgebaute Systeme bevorzugen, die es ihnen ermöglichen, (1) genau die Bausteine (Pro- dukte, Produktteile und Dienstleistungen) auszuwählen, die sie benötigen, und (2) das zu angemessenen und wettbewerbsfähigen Preisen. Die Auswertungen zeigen, dass Ultimate Luxury das am wenigsten bevorzugte Geschäftsmodellmuster ist. Während Mass Customization die höchste Bewertung erreicht, deuten die Daten darauf hin, dass die Kunden tendenziell preissensibel sind. Nichtsdestotrotz, wenn der Preis angemessen ist, bevorzugen Kunden individualisierbare Produkte und Dienstleistungen (Mass Customization) gegenüber der No-Frills-Option. Zur Erinnerung: Die No-Frills-Strategie ist zwar deutlich preisgünstiger als die Mass Customization, doch sie bietet keinerlei Individualisierungsmöglichkeiten.

Das Cluster Preisaufschläge zeigt, dass Kunden bereit sind, einen Preisaufschlag für modulare Produkte und Dienstleistungen zu bezahlen (Mass Customization), aber nicht für garantierte Verfügbarkeit. Interessant erscheint des Weiteren, dass Robin Hood sogar noch knapp besser abschneidet als die Solution-Provider-Strategie. In diesem Cluster wird sehr deutlich, dass die befragten Teilnehmer Endkunden sind. Eine

\section{Tab. 2 Ranking der Geschäftsmodellmuster}

\begin{tabular}{|c|c|c|c|}
\hline Cluster & Rang & Geschäftsmodellmuster & Score \\
\hline \multirow[t]{3}{*}{ Distribution } & $\# 1$ & Supermarket & 100 \\
\hline & $\# 2$ & Direct Selling & 73 \\
\hline & \#3 & Full-Service-Provider & 0 \\
\hline \multirow[t]{3}{*}{ Individualisierung } & $\# 1$ & Mass Customization & 100 \\
\hline & $\# 2$ & No Frills & 55 \\
\hline & \#3 & Ultimate Luxury & 0 \\
\hline \multirow[t]{4}{*}{ Preisaufschläge } & $\# 1$ & Mass Customization & 100 \\
\hline & \#2 & Robin Hood & 34 \\
\hline & \#3 & Solution Provider & 23 \\
\hline & $\# 4$ & Guaranteed Availability & 0 \\
\hline \multirow[t]{9}{*}{ Sonstige Preisstrategien } & $\# 1$ & Flatrate & 100 \\
\hline & $\# 2$ & Freemium & 91 \\
\hline & $\# 3$ & Cross-Selling & 90 \\
\hline & $\# 4$ & Hidden Revenue & 63 \\
\hline & \#5 & Pay-per-Use & 51 \\
\hline & \#6 & Add-on & 51 \\
\hline & $\# 7$ & Subscription & 46 \\
\hline & $\# 8$ & Self-Service & 31 \\
\hline & \#9 & Barter & 0 \\
\hline
\end{tabular}




\section{Kernthesen}

- Die Konkurrenz durch große internationale Plattformkonzerne und die zunehmende Bedeutung von Plattformen und Ökosystemen erfordern neue, strategisch abgestimmte Geschäftsmodelle

- Kundenvertrauen ist ein entscheidender Faktor für den Markterfolg von digitalen Produkten und Dienstleistungen und ist in der Geschäftsmodellauswahl zu berücksichtigen.

- Plattform- und Ökosystemgeschäftsmodelle, die das Kundenvertrauen berücksichtigen, sind nachhaltiger als hauptsächlich auf die Monetarisierung von Kundendaten setzende

Untersuchung im Business-to-Business-Kontext hätte mit an Sicherheit grenzender Wahrscheinlichkeit signifikant andere Ergebnisse erbracht.

Aus den Ergebnissen für den Cluster Sonstige Preisstrategien fällt direkt auf, dass die Befragten das Flatrate-Muster beinahe so stark favorisieren wie die Muster Freemium und CrossSelling. Weitere herausragende Ergebnisse in diesem Cluster sind die Rankings der Geschäftsmodelle Hidden Revenue und Barter. Während den Befragten das Tauschgeschäftsmodellmuster als am wenigsten attraktiv erscheint, ist Hidden Revenue für sie akzeptabel. Es ist hervorzuheben, dass beim Tauschgeschäftsmodellmuster (Barter) keine monetäre Bezahlung stattfindet. Unternehmen stellen hier Produkte und Dienstleistungen kostenlos zur Verfügung und dürfen im Gegenzug Kundendaten verarbeiten. Das Geschäftsmodell der versteckten Einnahmen (Hidden Revenue) funktioniert ganz ähnlich. So werden etwa Kundendaten genutzt, um personalisierte Werbung anzuzeigen. Allerdings wird dieser Vorgang dem Kunden weniger explizit gemacht. Entsprechend bestätigt sich auch hier das bereits erwähnte und aus anderen Studien [2,3] bekannte, teilweise paradoxe Verhalten von Kunden hinsichtlich der Verarbeitung ihrer privaten Daten. Ein weiteres, überraschendes, aber hierzu passendes Ergebnis ist, dass in unserer Untersuchung das Geschäftsmodellmuster Hidden Revenue besser bewertet wird als Pay-per-Use. Bei Pay-per-Use bezahlen Kunden nur, was sie nutzen, sodass dieses Preismodell deutlich fairer sein müsste als andere Geschäftsmodellmuster. Dennoch bevorzugen die Kunden offenbar die Verarbeitung ihrer Daten im Hintergrund oder den Verkauf ohne ihre ausdrückliche Zustimmung (Hidden
Revenue) zur Erlangung kostenloser Produkte und Dienstleistungen. Letztlich zeigen die Ergebnisse dieses Clusters zusammengefasst, dass Manager insbesondere die Flatrate-, Freemium- oder Cross-Selling-Geschäftsmodellmuster in Betracht ziehen sollten. Wenn keines dieser drei Muster umgesetzt werden kann, wäre es hilfreich, auf die am nächstbesten bewerteten Muster zuzugreifen.

\section{Fazit und Ausblick}

Die zunehmende Bedeutung von digitalen Plattformen und Ökosystemen, verbunden mit technologischen Entwicklungen wie dem Internet der Dinge, erfordern von Unternehmen eine stärkere Kundenorientierung als bisher. Im Spannungsfeld zwischen der notwendigen Wertschöpfung für das Unternehmen, der begrenzten Zahlungsbereitschaft der Kunden für Produkte und Dienstleistungen und der Notwendigkeit, das Kundenvertrauen zu gewinnen und zu erhalten, sind neue Geschäftsmodelle erforderlich. Unter der Prämisse, dass eine Berücksichtigung der Kundenbedürfnisse und -präferenzen in der Geschäftsmodellauswahl von Unternehmen eine nachhaltig erfolgreiche Unternehmensstrategie sein kann, bietet dieser Beitrag praxisrelevante Hinweise, die vor allem für kleine und mittelständische Unternehmen wertvoll sein können.

Mittels einer strukturierten Methodik, die Erkenntnisse zu Geschäftsmodellen aus verwandten wissenschaftlichen Arbeiten mit einer empirischen Untersuchung kombiniert, ergibt sich durch eine Experten- und Nutzerbefragung ein Ranking von für Plattformen und Ökosysteme geeigneten Geschäftsmodellmustern, das die Kundenakzeptanz für verschiedene Geschäftsmodelle für IoT-Produkte und -Dienstleistungen widerspiegelt. Die Ergebnisse der Studie liefern direkte Hinweise darauf, welche Geschäftsmodelle aus Kundenperspektive akzeptabel sind oder nicht. Damit ergibt sich für Manager die Möglichkeit, diese Erkenntnisse direkt in der Praxis umzusetzen. Die Praxisumsetzung wird auch dadurch erleichtert, dass ein direkter Bezug der präsentierten Cluster zum aus strategischer Sicht wichtigen Marketingmixinstrument hergestellt wird. Die Ergebnisse der präsentierten Studie bieten somit eine Hilfestellung für Unternehmen, die eine kundenfreundliche und nachhaltig erfolgreiche Produkt-, Preis- und Distributionspolitik anstreben.

Die Ergebnisse der Studie sollen zukünftig in den sich in der Entwicklungsphase befindlichen EPN - Ecosystem Participation Navigator [7] einfließen und somit interessierten Unternehmen zugänglich gemacht werden. Der EPN ist eine von den Autoren erarbeitete Methodik mitsamt einfach zu 
bedienendem Online-Tool, die Unternehmen bei der Entwicklung von Geschäftsmodellen für digitale Ökosysteme unterstützt. Er richtet sich insbesondere an Unternehmer oder Entscheidungsträger, die ihr Start-up, ihr bereits etabliertes Unternehmen oder ihren Unternehmensbereich aktiv an den Wertschöpfungsprozess in einem digitalen Ökosystem einbinden wollen und dafür ein neues Geschäftsmodell benötigen. Derzeit basiert der EPN noch auf Vorarbeiten aus einem anwendungsorientierten Forschungsprojekt sowie einer ersten Expertenbefragung und soll zukünftig um die Ergebnisse der hier vorgestellten Studie ergänzt werden. Das OnlineTool und die Methodik sind jedoch bereits jetzt nutzbar über https://s.fhg.de/EPN-Tool.

Funding. Open Access funding enabled and organized by Projekt DEAL.

Open Access Dieser Artikel wird unter der Creative Commons Namensnennung 4.0 International Lizenz veröffentlicht, welche die Nutzung, Vervielfältigung, Bearbeitung, Verbreitung und Wiedergabe in jeglichem Medium und Format erlaubt, sofern Sie den/die ursprünglichen Autor(en) und die Quelle ordnungsgemäß nennen, einen Link zur Creative Commons Lizenz beifügen und angeben, ob Änderungen vorgenommen wurden.

Die in diesem Artikel enthaltenen Bilder und sonstiges Drittmaterial unterliegen ebenfalls der genannten Creative Commons Lizenz, sofern sich aus der Abbildungslegende nichts anderes ergibt. Sofern das betreffende Material nicht unter der genannten Creative Commons Lizenz steht und die betreffende Handlung nicht nach gesetzlichen Vorschriften erlaubt ist, ist für die oben aufgeführten Weiterverwendungen des Materials die Einwilligung des jeweiligen Rechteinhabers einzuholen.

Weitere Details zur Lizenz entnehmen Sie bitte der Lizenzinformation auf http://creativecommons.org/licenses/by/4.0/ deed.de.

\section{Literatur}

[1] Teece, D. J. (2018). Business models and dynamic capabilities. Long Range Planning, 51(1), 40-49.

[2] Mihale-Wilson, C., Zibuschka, J., \& Hinz, O. (2019). User preferences and willingness to pay for in-vehicle assistance. Electronic Markets, 29(1), 37-53.

[3] Zibuschka, J., Nofer, M., Zimmermann, C., \& Hinz, O. (2019). Users' preferences concerning privacy properties of assistant systems on the Internet of things. In Proceedings of the Twenty-fifth Americas Conference on Information Systems (AMCIS). Cancun. Mexico.

[4] Gassmann, O., Frankenberger, K., \& Csik, M. (2017). Geschäftsmodelle entwickeln. 55 innovative Konzepte mit dem St. Galler Business Model Navigator. München: Hanser.

[5] Borden, N. H. (1964). The concept of the marketing mix. Journal of Advertising Research, 4(2), 2-7.

[6] Flynn, T. N., Louviere, J. J., Peters, T. J., \& Coast, J. (2007). Bestworst scaling: what it can do for health care research and how to do it. Journal of Health Economics, 26(1), 171-189.

[7] Mihale-Wilson, C., \& Kubach, M. (2019). Ecosystem Participation Navigator: Positionierung und Geschäftsmodellierung in digitalen Ökosystemen. In S. von Engelhardt \& S. Petzolt (Hrsg.), Das Geschäftsmodell-Toolbook für digitale Ökosysteme (S. 80-93). Frankfurt/Main: Campus. 